\title{
Mircea Eliade, modes et modèles culturels
}

Occultisme, ésotérisme, magie, rituels initiatiques, mythes...

\section{Eugen Simion}

\section{(2) OpenEdition}

\section{Journals}

Édition électronique

URL : https://journals.openedition.org/rbnu/3215

DOI : $10.4000 /$ rbnu. 3215

ISSN : 2679-6104

Éditeur

Bibliothèque nationale et universitaire de Strasbourg

\section{Édition imprimée}

Date de publication : 1 novembre 2011

Pagination : 68-75

ISSN : 2109-2761

Référence électronique

Eugen Simion, « Mircea Eliade, modes et modèles culturels », La Revue de la BNU [En ligne], 4 | 2011 mis en ligne le 01 novembre 2011, consulté le 08 août 2021. URL : http://journals.openedition.org/ rbnu/3215; DOI : https://doi.org/10.4000/rbnu.3215 Attribution - Pas d'Utilisation Commerciale - Partage dans les Mêmes Conditions 4.0 International. 


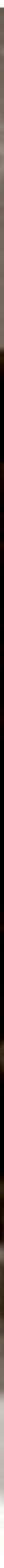

Mircea Eliade

(coll. Bibliothèque de l'Académie roumaine, cabinet des estampes) 


\section{MIRGEA ELIADE, MODES ET MODÈLES CULTURELS : occultisme, ésotérisme, magie, rituels initiatiques, mythes...}

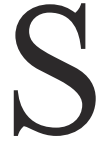

'il est une personne qui a étudié en profondeur et sur une très longue période au $20^{\mathrm{e}}$ siècle - appelé par lui-même le siècle " des sciences réductionnistes " - les phénomènes d'occultisme, d'ésotérisme ou de magie, c'est bien Mircea Eliade. Né en 1907, Mircea Eliade fit ses études universitaires à Bucarest. En 1928, il partit avec une bourse en Inde où il resta trois ans. Il y étudia le sanscrit, le chamanisme et le yoga, bref il y découvrit la spiritualité hindoue dans sa globalité. En 1931, il retourna en Roumanie et, à partir de son expérience asiatique, il publia des journaux intimes (comme Chantier, en 1935), des romans à thèmes hindous (La nuit bengali, en 1933) et, parallèlement, des études sur la spiritualité hindoue (un traité, par exemple, intitulé L'alchimie asiatique, en 1935 ; ou encore Yoga. Essai sur les origines de la mystique indienne, en 1936, entre autres). Dès cette époque, il orienta ses efforts de création dans deux directions : l'histoire des croyances et des idées religieuses d'une part, et la littérature de fiction, et particulièrement le roman, d'autre part. Deux idées, deux chemins intellectuels que Mircea Eliade a respectés et suivis toute sa vie. Son œuvre scientifique, écrite en anglais et en français, est connue. C'est elle qui l'a rendu célèbre, surtout après qu'il se fut établi, vers le milieu des années cinquante, aux Etats-Unis, plus précisément à l'université de Chicago, où il dirigea jusqu'à la fin de sa vie le département d'Histoire des religions et où il publia un nombre impressionnant d'écrits sur les mythes, le sacré et le profane, l'histoire des croyances religieuses, ou encore les symboles. L'autre domaine, celui de la littérature de fiction, est moins connu. Ecrits en roumain, ses romans et ses nouvelles sont connus et appréciés dans son pays d'origine, la Roumanie et, dans une certaine mesure, grâce aux traductions, en France, en Allemagne et dans quelques pays d'Europe du Sud-Est, comme la Serbie. On peut également inclure dans le domaine de la littérature les journaux intimes d'Eliade, traduits en partie en français. Son intérêt pour ce genre subjectif peut être comparé, au $20^{\mathrm{e}}$ siècle, à celui de son ami Ernst Jünger et, dans une certaine mesure, à celui de Julien Green.

Quelle place occupent les thèmes que j'ai annoncés dans le titre de cet article (l'occultisme, l'ésotérisme, la magie ou les rituels initiatiques) dans cette immense œuvre scientifique et littéraire, y compris dans l'activité de diariste d'Eliade ? Il s'agit, comme on le constatera, d'une place qui, sans être essentielle ou prioritaire, n'en est pas moins importante, d'un domaine qu'il n'a pas ignoré quand il s'est efforcé d'étudier les cultures européennes et non-européennes et d'élaborer ainsi une histoire des idées et des croyances religieuses. Sur la magie, sur la sorcellerie, sur les mystères et les techniques d'initiation, sur les pratiques occultes et les diverses formes d'ésotérismes européens et non-européens, Eliade a écrit à maintes reprises. On peut même avancer qu'il prend tout au sérieux, qu'il veut comprendre non seulement la structure formelle des symboles, mais aussi leur signification profonde, interne. Il réussit à nous convaincre que la sorcellerie n'est pas simple affaire de charlatans, bien qu'elle puisse l'être aussi, que la sorcellerie, la magie et toutes les pratiques qui font partie de cette famille ont une dimension religieuse ; elles représentent quelque chose d'essentiel pour l'homme archaïque et même pour l'homme moderne, désacralisé, terrorisé par l'Histoire. C'est de cette idée qu'Eliade part quand il veut expliquer les mystères qui ont toujours attiré l'homme et 


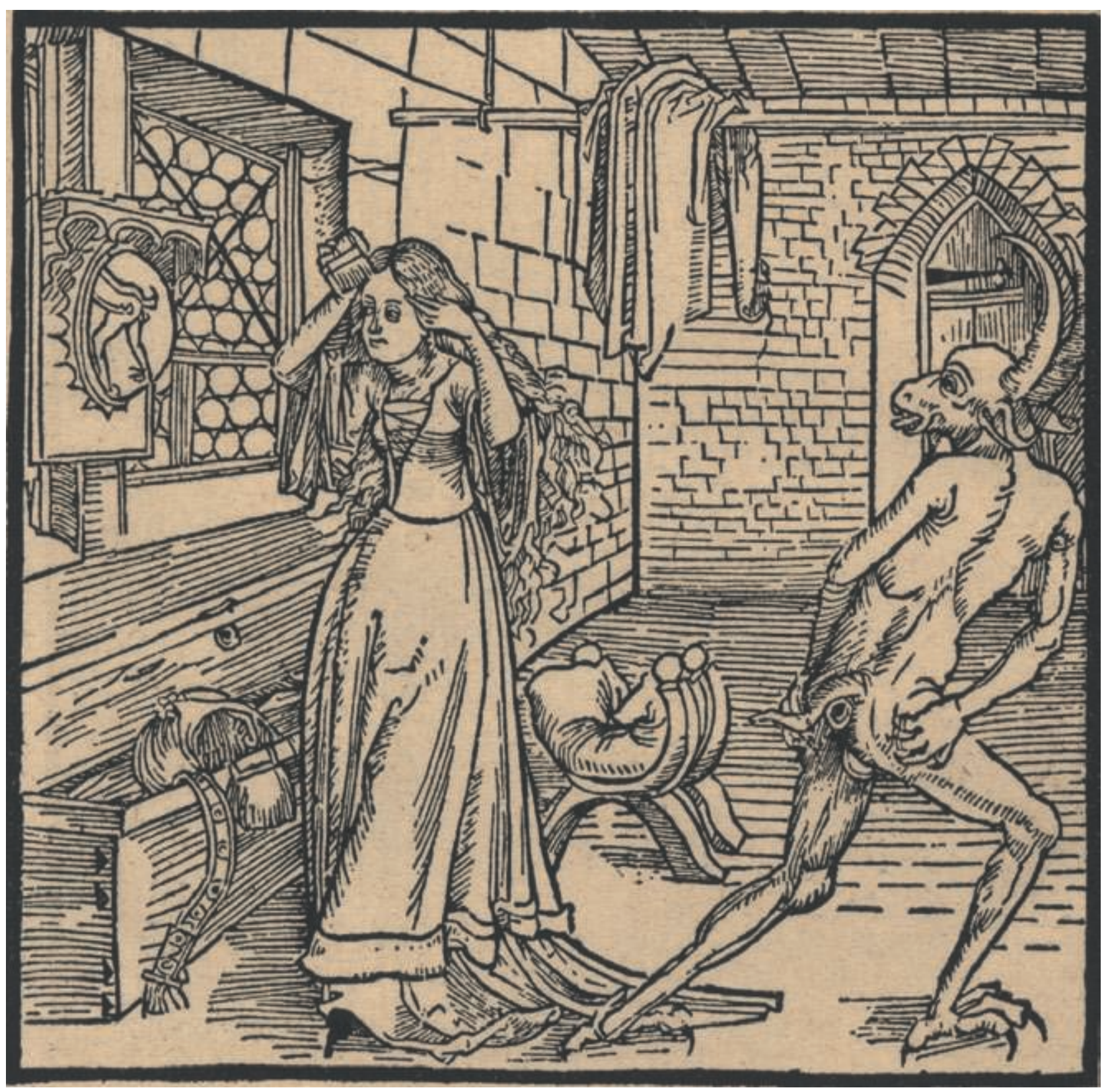

Latour Landry, Geoffroy de. - Der Ritter vom Turn, extrait de l'incunable publié à Bâle en 1493 (édition en fac-similé, Munich, 1922 ; coll. Bibliothèque de l'Académie roumaine) 
qui l'ont amené plusieurs fois à croire et à se laisser séduire par ce qu'il ne voit pas et qu'il ne touche pas avec les doigts de son esprit. Eliade parle en érudit dans presque tous ses livres de cette part de mystère qui est inhérente à tout être humain et qu'il appelle du terme générique de dimension du sacré ou, pour le dire plus simplement, de cette attirance pour le sacré qui se trouve dans chaque individu, quelle que soit sa condition.

L'idée de sacralité, inhérente donc à l'être

humain, rejoint, dans la pensée d'Eliade, celle de la permanence du mythe. L'homme est une création de mythes, dit-il, il n'est pas une totalité de complexes, comme le dit la psychanalyse, ni une totalité de relations sociales, ainsi que le soutiennent les marxistes, ni non plus un être de papier, comme l'affirment les structuralistes. Il est avant tout une totalité de mythes qu'il porte en soi, sans le savoir, jusqu'à la mort. Et la mort même est entourée de mythes auxquels l'homme se réfère et qu'il veut dominer au moyen de diverses pratiques spirituelles. L'homme, observe Eliade, est entouré de signes, de miracles, d'épiphanies. Il est la proie d'étranges oublis. L'homme moderne cherche toujours quelque chose qu'il ne connaît pas et que la plupart du temps il ne trouve pas. Or ce quelque chose peut être essentiel pour lui, car son amnésie cache un grand secret de son être. Selon Eliade, l'homme moderne (l'homme des "sciences réductionnistes") n'est pas aussi pauvre et vide qu'on veut bien le dire : il est plus riche et plus complexe. Il porte en lui des mythes, des archétypes et il a la capacité de perfectionner son être en retrouvant ces modèles culturels. Dans une note du Journal de juin 1960, Eliade, qui est en général très modeste et ne fait pas cas de ses découvertes, commente très clairement sa contribution à la resacralisation de la nature et aussi à l'importance de la dimension spirituelle de l'homme moderne, en proie aux vicissitudes de l'Histoire : " La nature ", dit-il, " a été désacralisée et vidée de ses significations culturelles et finalement philosophiques, comme le dit Goethe. Je crois pouvoir dire que je me trouve parmi les rares Européens qui ont réussi à revaloriser la nature, en mettant au jour les hiérophanies et la structure de la religiosité cosmique. Cela me semble d'autant plus important qu'à la différence de Nietzsche, par exemple, qui essaie de retrouver la nature au moyen des techniques et des valeurs culturelles - Dionysos est avant tout un sujet de philosophie classique - on est parvenu à la redécouverte des sacralités cosmiques en méditant l'expérience quotidienne des paysans roumains ou bengalais. On a commencé, donc, par des situations historiques contemporaines et des valeurs culturelles vives..."

Dans Modes culturelles et histoire des religions (1965), Eliade conteste la justification donnée par les existentialistes au phénomène d'aliénation de l'homme, à savoir l'excessive préoccupation de ceux-ci pour la " situation " de l'homme et son engagement historique - phénomène d'ailleurs réel, tragique - et il soulève à nouveau des questions à propos de la vogue structuraliste. D'après lui, l'homme n'est pas une structure simple et vide et, d'une façon générale, dans le monde archaïque comme dans le monde moderne, il existe un scénario mythico-rituel, par lequel une réalité spirituelle profonde s'exprime. Si l'homme moderne est en crise - et c'est bien le cas - cela est dû au fait qu'il a oublié d'où il vient et les sciences positives ne l'aident certes pas à le redécouvrir. Bref, l'anthropologie qu'Eliade veut instaurer envisage la possibilité d'un accès de l'homme moderne à ce " monde auroral ", ainsi que celle de le sauver grâce à l'expérience du sacré.

Il s'agit là pour Eliade d'une contribution essentielle à ce siècle dans lequel l'homme a perdu, entre autres, jusqu'à l'unité de son image dans le monde. On doit lui savoir gré du fait que dans un siècle de totalitarismes sanglants, où les systèmes idéologiques se contestent sauvagement, il voit l'homme comme un être capable de reconquérir le sens de la globalité du monde et de vaincre les violences de l'Histoire. Comment ? En redécouvrant les mythes et plus généralement en s'effor- 


\section{Tout se rejoint, les jeunes hippies qui se promènent sales et déguenillés ne font que reproduire, sans le savoir, les jeunes cyniques d'il y a à peu près deux mille ans, qui, par leur comportement, défiaient les institutions et les mentalités de leur époque.}

çant de comprendre le rôle et les fonctions culturelles des mystères et des signes qui accompagnent l'homme partout et dans toutes les étapes de son existence. Dans une conférence tenue à Philadelphie en mai 1974 et publiée dans le livre Occultisme, sorcellerie et modes culturelles (The University of Chicago Press, 1976), Eliade étudie, plus systématiquement, les formes d'occultisme et d'ésotérisme dans le monde moderne qui représentent, note-t-il dès le début, de façon idéale et souvent utopique l'essai de " restauration mystique de la dignité et des pouvoirs originaux de l'homme ". Dans certains cas, la résurgence des pratiques occultes et ésotériques représente aussi une forme de révolte des artistes, quand ils adoptent dans leur art ces techniques d'initiation, une forme de révolte, dis-je, contre les institutions et l'idéologie du monde bourgeois, ainsi qu'on peut le voir de Baudelaire aux surréalistes. Et, comme il le fait toujours, Eliade cherche les sources de ce phénomène qui a resurgi après 1960 dans le monde occidental. Pour lui, cela signifie un retour au passé, aux mythes et aux archétypes de la Renaissance italienne, par exemple, quand la magie et l'ésotérisme hermétique ont joué un rôle essentiel dans la restructuration de l'art. Sa conclusion est la suivante : "Les recherches contemporaines ont dévoilé la signification religieuse profonde et la fonction culturelle d'un grand nombre de pratiques, croyances et théories occultes, communes à beaucoup de civilisations aussi bien européennes que non européennes, et à tous les niveaux de la culture, en commençant par les rites folkloriques - comme la magie et la sorcellerie - jusqu'aux techniques secrètes et aux spéculations ésotériques les plus savantes et les plus élaborées : l'alchimie, le yoga, le tantrisme, le gnosticisme, l'hermétisme de la Renaissance, les sociétés secrètes et les loges maçonniques de la période des Lumières ". Tout ceci, conclut Eliade, ne constitue pas des actes isolés. Ils ont " une structure religieuse " et renvoient aux pratiques orgiaques réelles ou imaginaires du passé. Tout se rejoint, les jeunes hippies qui se promènent sales et déguenillés ne font que reproduire, sans le savoir, les jeunes cyniques d'il y a à peu près deux mille ans, qui, par leur comportement, défiaient les institutions et les mentalités de leur époque.

Et la littérature ? Que fait-elle des mystères, de la magie, de l'occultisme, de l'ésotérisme ou des mythes ? D'après Eliade, cela ne pose aucun problème. Bien plus : tous les phénomènes cités peuvent ressusciter la littérature moderne qui passe, de façon évidente, par une étape de décadence. Quelques-uns disent qu'elle se prépare même à mourir, et que ce fait n'est pas propre à notre époque ou à celle d'hier mais qu'il remonte à bien longtemps. Eliade n'est pas de cet avis, il croit seulement que la redécouverte des mythes et des phénomènes liés à la structure du mythe offrira une nouvelle chance à la littérature profane. Un phénomène double, donc : le mythe resacralise l'homme profane, et la littérature, à partir de ce nouveau miracle, fondamental pour le monde de la modernité, échappera à la médiocrité, à la sécheresse et au vide des valeurs dans lesquels l'a plongée la terreur de l'Histoire. Eliade a soutenu cette idée avec une cohérence éblouissante, à une époque où les écoles formalistes en matière de critique étaient au pouvoir en Occident 


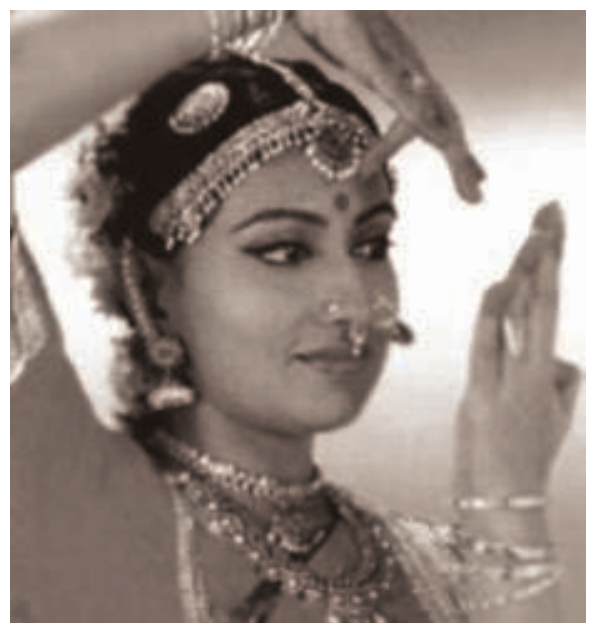

La danse sacrée (détail) (coll. Bibliothèque de l'Académie roumaine, cabinet des estampes)

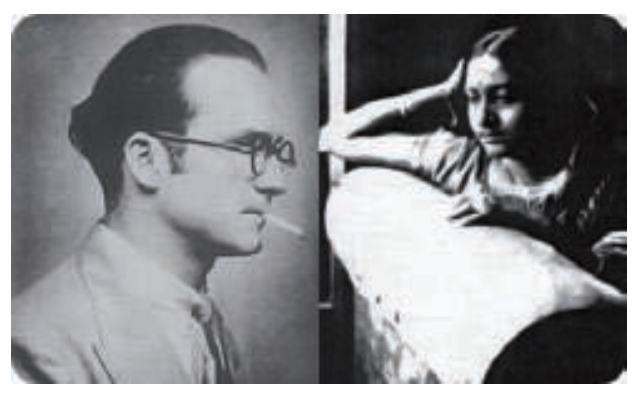

Mircea Eliade et Maitreyi Devi, inspiratrice du roman Maitreyi qu'Eliade publie en 1933 (coll. Bibliothèque de l'Académie roumaine, cabinet des estampes) et où le nouveau roman dominait la théorie littéraire et même la pratique littéraire occidentales. Il n'a pas été écouté à son époque et je me demande s'il est lu et entendu aujourd'hui. Ce qu'il y a de sûr c'est que, dès sa jeunesse, sa prose s'inspire des mystères qu'Eliade a analysés dans ses études sur les modèles culturels. Il faut ici faire une parenthèse : Eliade a toujours soutenu que le modèle européen n'avait plus, dans le monde moderne, la primauté en matière de vie spirituelle. Il n'est plus, de la même manière, le seul modèle créateur. Il y en a d'autres, venus de l'extérieur de l'espace européen, qui offrent de nouvelles perpectives, qui sont créatifs. Cette idée a valu à Eliade la réputation d'être un esprit anti-occidental. Il n'en est rien : Eliade n'est pas hostile à l'Occident spirituel, mais c'est en toute lucidité qu'il a prévu, avec de l'avance sur nous, que l'Occident européen n'est plus le seul modèle culturel dans la planète de l'esprit. C'est le rôle de la marginalité de donner des modèles. L'explosion du roman sud-américain dans la dernière moitié du $20^{\mathrm{e}}$ siècle nous montre qu'il ne s'est pas trompé. Eliade, je l'ai dit, parle dans son œuvre savante des mythes, des techniques d'initiation, des épiphanies, des mondes parallèles et des coïncidences mystérieuses et, parallèlement, il écrit dans ses romans et dans ses récits sur les mêmes sujets, en opposition complète avec la pratique et la théorie littéraires de son époque. J'ai déjà analysé la prose de Mircea Eliade dans un de mes livres. Je veux me limiter ici à expliquer brièvement la manière dont Eliade utilise ce que l'on peut appeler les mystères dans sa prose. Après avoir écrit, dans les années trente, un roman de type existentialiste sous l'influence de Gide (Retour du paradis ; Les hooligans), il avait essayé d'écrire un roman avec Joyce pour modèle (La lumière qui s'éteint, en 1934)... Il avait essayé aussi d'utiliser, je l'ai déjà signalé, son expérience existentielle et spirituelle en Inde dans deux romans (Isabelle et les eaux du diable, en 1930, et La nuit bengali, en 1933), où les techniques du journal intime sont essentielles. A partir de 1936, Eliade s'oriente vers un autre type de narration (Mademoiselle Christine, Le Serpent) où la magie et ce que l'auteur appelle " une réalité irrationnelle mais concrète " constituent des points de référence fondamentaux. Il s'agit d'une " intuition fantastique " - comme l'appelle le prosateur dans un article publié dans un volume d'essais, Océanographie, en 1934 - une intuition qui permet à l'esprit créateur d'inclure " globalement et essentiellement la réalité " et une expérience " où s'est concentrée l'intuition globale de la vie et de la mort ". Dans Mademoiselle Christine, il parle de fantômes, mais d'une autre manière et avec d'autres effets que dans le roman noir. Le prosateur s'intéresse ici au mode dans lequel l'irrationnel se glisse dans l'existence et dans la pensée des hommes rationnels, à l'angoisse mais aussi à la mythologie séduisante qu'il produit. Le personnage central est ici non le fantôme lui-même, mais une fillette possédée par le diable et qui en est le messager. Le serpent (1937) évoque le phénomène de séduction (magique et érotique) par lequel passe un groupe de jeunes de $\mathrm{Bu}$ carest dans une forêt proche de la ville. Des superstitions, de la magie ? Ces éléments existent dans le récit d'Eliade, mais le mystère reste jusqu'à la fin sans solution. Il s'agit là d'un thème et d'un procédé narratif 
qu'Eliade utilisera aussi dans sa prose ultérieure. Dans Minuit à Serampore, trois Européens sceptiques sont soumis à une opération magique par le professeur Budge de Calcutta, adepte des pratiques tantriques. Ils deviennent les témoins d'un événement vieux de 150 ans. Une rupture, une sortie du temps et de l'espace ; un retour donc vers le passé. Comment l'expliquer ? Cela ne peut se faire rationnellement. Le sage Swami Shivanananda de l'Himalaya est même capable de confirmer la logique du magique.

Le même schéma narratif est utilisé par Eliade dans le petit roman Le secret du docteur Honigberger (1940) et également, après qu'il fut parti en exil en France puis aux États-Unis, dans ce que les critiques littéraires ont appelé les récits " mythiques ", écrits après la guerre : le roman Forêt interdite, la nouvelle Chez les Tziganes, le petit roman Le vieil homme et l'officier, les récits Les trois grâces, Une photographie vieille de quatorze ans, Mille deux cents têtes de boufs en sont des exemples. Il n'y a pas lieu de les présenter tous ici, avec leurs mystères, et les mythes et les symboles qui fonctionnent dans l'ombre. Ce qui se répète dans ces œuvres, c'est ce qu'Eliade appelle lui-même, en s'appuyant sur les philosophes des sciences occultes, "une série d'existences mutuellement contradictoires " et un nombre de mythes qui se révèlent dans la prose médiocre de la vie, sans que, comme nous l'avons déjà dit, les personnages ne s'en rendent compte. L'enseignant Fărâmă du Vieil homme et l'officier vit le mythe de Shéhérazade dans un régime politique totalitaire : en s'intéressant à un ancien élève, Fărâmă devient suspect à la police politique de l'époque du stalinisme et pour lui échapper il doit inventer chaque jour un nouveau conte à partir d'une histoire qu'il complique inlassablement. Il mélange les plans temporels et imaginaires, il raconte des événements d'autrefois, devenant ainsi de plus en plus bizarre aux yeux de ses enquêteurs qui sont des esprits rationnels. A la fin, quand le vieux professeur paraît avoir fui loin d'eux, lui qui semble savoir " beaucoup de choses " est arrêté par d'autres individus intéressés par ces mystères. La Shéhérazade du régime bolchevique va donc connaître des nuits et des jours très difficiles.

Dans un autre conte, Les trois grâces, Eliade fait apparaître Perséphone, une Perséphone tragique, poursuivie elle aussi par la police politique, et dans Le temps d'un centenaire (1978), il illustre narrativement l'idée de la régénération biologique et du temps post-historique, un thème proche de la littérature de science- fiction. Eliade le place dans le champ plus suggestif de la littérature mythique et il le rapproche d'un autre thème, essentiel dans toute sa prose, celui de la rupture temporelle, du passage imperceptible d'un temps à un autre. L'idée acquiert de la cohérence et de l'expressivité dans la plus belle nouvelle qu'ait écrite Eliade : Chez les Tsiganes, un chef-d'œuvre en matière de style. Son histoire, qui part de l'idée que le mystère se cache dans l'Histoire, c'est-à-dire dans la réalité profane, repose sur un procédé en deux étapes, à deux vitesses : il s'agit d'abord de la découverte du mystère tel qu'il se donne à voir sous forme de signes, d'oublis, de preuves d'irrationalités concrètes et surtout de coïncidences étranges, de ruptures dans la chronologie du temps, bref, le moment où le mystère se révèle et, en deuxième lieu, l'effet même du mystère sur l'existence d'un quelconque individu. Par rapport à ce phénomène, les personnages d'Eliade se divisent en deux catégories : d'une part ceux qui ne croient à rien de ce qui se passe sous leurs yeux ou des événements qu'on leur raconte. Ils représentent les esprits étroitement rationalistes, les représentants de l'ordre public ou de la police politique, personnifiés par exemple par les enquêteurs du Vieil homme et l'officier; d'autre part ceux qui, comme le professeur retraité Fărâmă ou le professeur Gavrilescu dans la nouvelle Chez les Tsiganes, vivent ces phénomènes sans trop savoir de quoi il s'agit ni ce qui se passe. Leur rôle est de les raconter à l'infini, en mélangeant les faits, en les relativisant à mesure qu'ils les relatent. Il y a conflit entre ces deux catégories, sans que l'auteur donne explicitement raison à l'une ou à l'autre et sans qu'il donne, à la fin, une explication claire de la thématique présentée. Il ne fait en réalité que la présenter et dit à son lecteur : débrouille-toi tout seul...

Ce scénario est présenté sous forme d'essai dans le conte Une photographie vieille de quatorze ans où il s'agit d'un homme simple, un paysan du Danube qui, arrivé dans un pays de langue anglaise, prétend que le docteur Martin, prédicateur à la Basilique du Salut, a guéri par télépathie sa femme, Thècle, qui était asthmatique. De plus, Thècle se met à ressembler, après sa guérison, à une photo d'elle vieille de quatorze ans. Elle aurait donc rajeuni. Dumitru, le paysan du Danube, croit sincèrement à ce miracle. Quand il revient, quatre années plus tard, à la Basilique du Salut, il apprend que le docteur Martin a été convaincu d'escroquerie et qu'il a été arrêté pendant un temps. Depuis sa libération, il pratique un métier honorable : 
il est serveur dans un restaurant. Il ne croit plus aux miracles. Dieu, dit-il, soutenu en cela par de jeunes spécialistes de bouddhisme Zen, de sémiologie et de sociologie, est mort ou agonise. En tout cas, Il ne fait plus partie du monde. Opinion que Dumitru, dans sa naïveté, rejette. Elle lui paraît même scandaleuse, inadmissible, car si Dieu est mort ou qu'Il agonise, quel est alors le rôle de l'homme sur la terre ? Ce conte, qui n'est pas une réussite au plan esthétique, a le mérite de formuler clairement la thématique de l'écrivain, à savoir le retrait de la divinité du monde et de suggérer, au moyen d'une fiction narrative, un cas de renaissance spirituelle chez un individu simple. Dumitru a fui la Roumanie d'après-guerre : son geste, évidemment politique, peut aussi avoir une autre signification. Il a fui un système politique répressif, mais il a apporté avec lui, dans la sceptique Amérique, dans la civilisation occidentale désacralisée, une croyance, et donc un type de culture dans laquelle Dieu n'est pas laissé "à l'agonie ". L'homme du Danube ne peut admettre ce désastre cosmique. Dumitru, le naïf, l'immigré, est porteur d'une grande vérité dont la révélation devrait faire honte au sophiste qu'est Lucio et à ses amis philosophes entachés de scepticisme.

Oui, Eliade introduit dans ses histoires les phénomènes cachés, mystérieux dont nous avons parlé. Mais ses mises en garde concernant l'ésotérisme et l'occultisme sont claires. Il n'est pas intéressé par le côté ésotérique et d'une façon générale il n'a pas de goût pour les choses obscures. Guénon n'est pas son modèle. Dans Fragments d'un journal, il écrit : "Dans tout ce que j'ai pu écrire, même dans mes livres d'histoire des religions et de philosophie de la culture, il me manque le goût de l'obscur ". Eliade utilise pourtant dans ces contes des éléments occultes, des irrationalités concrètes, comme nous l'avons déjà dit, grâce auxquelles il ouvre une fenêtre vers un monde de signes, un monde où cohabitent des plans parallèles de temps et d'espace, un monde dans lequel les épiphanies continuent à exister. Que faut-il comprendre ? Qu'Eliade le romancier ne veut pas obligatoirement illustrer d'une manière orthodoxe et avérée les phénomènes que l'historien des religions étudie. Il a protesté plusieurs fois contre cette analogie. La dernière fois, il l'a fait dans une lettre qu'il m'a envoyée au moment où je me préparais à publier à Bucarest sa prose fantastique (en 1981).

Je me suis demandé alors - et je le fais encore au- jourd'hui - si on peut séparer l'Eliade spécialiste d'histoire des religions de l'Eliade romancier du fantastique. Il est sûr qu'il ne cherche pas à cultiver, en prose, la magie pour elle-même, mais plutôt le sentiment du magique et de ce que la magie peut provoquer dans l'existence d'un homme simple. Comme, par exemple, le fait qu'une cantatrice quelconque des brasseries bucarestoises devienne, sans le savoir, la messagère d'un Orphée local dont elle est amoureuse dans À la cour de Dionis ; une Eurydice qui, par son chant, transmet le message d'un Orphée oublié. Redisons-le, l'imaginaire d'Eliade part du réel, mais chez lui le " réel " comporte sa part de mystère, il est toujours envahi de symboles et d'éléments irrationnels, ésotériques, occultes, anormaux ou paranormaux, de choses et d'événements qu'on ne peut pas expliquer mais, que nous y croyions ou que nous n'y croyions pas, nourrissent notre imaginaire et que nous portons tous en nous... Tout ceci, suggère Mircea Eliade - l'homme du procès, comme a dit de lui son ami Cioran, ou le spécialiste en histoire des religions qui n'est pas religieux, ainsi que le définit un autre ami, Eugène Ionesco - vient de temps immémoriaux dans l'imaginaire de l'homme moderne, de très loin, de l'époque au cours de laquelle les mythes ont été créés. Des mythes que l'homme contemporain vivant dans des sociétés désacralisées, comme c'est le cas aujourd'hui, continue de véhiculer souvent sans le savoir. Voici, brièvement, le testament d'Eliade. C'est le modèle culturel qu'il a voulu expliquer dans la littérature de son siècle sans être toutefois ni vraiment écouté ni vraiment suivi.

\section{Eugen Simion}

\section{Notes}

1 - Mircea Eliade est mort en 1986.

2 - Aux éditions Gallimard. 